\title{
Assessing the Contribution of Bioeconomy to the Total Economy: A Review of National Frameworks
}

\author{
Stefania Bracco ${ }^{1, *}$, Ozgul Calicioglu ${ }^{1,2}$, Marta Gomez San Juan ${ }^{1}$ and Alessandro Flammini ${ }^{1}$ \\ 1 Food and Agriculture Organization of the United Nations, Viale delle Terme di Caracalla, \\ 00153 Roma RM, Italy; Ozgul.Calicioglu@fao.org (O.C.); Marta.GomezSanJuan@fao.org (M.G.S.J.); \\ Alessandro.Flammini@fao.org (A.F.) \\ 2 Department of Civil and Environmental Engineering, The Pennsylvania State University, \\ 212 Sackett Building, University Park, PA16802, USA \\ * Correspondence: stefania.bracco@fao.org or stefania.bracco2@gmail.com; Tel.: +39-065-705-5955
}

Received: 13 April 2018; Accepted: 17 May 2018; Published: 23 May 2018

\begin{abstract}
Developments in technology have enabled envisioning the derivation of materials and products from renewable biomass as an alternative to finite fossil-based resource consumption. Therefore, bioeconomy is regarded as an opportunity for sustainable economic growth. Countries are formulating strategies in accordance with their goals to attain a bioeconomy. Proper measurement, monitoring, and reporting of the outcomes of these strategies are crucial for long-term success. This study aims to critically evaluate the national methods used for the measurement, monitoring, and reporting of bioeconomy contribution to the total economy. For this purpose, research and surveys have been conducted on selected countries (Argentina, Germany, Malaysia, the Netherlands, South Africa, and the United States). The results reveal that the bioeconomy targets set up in the strategies often reflect the country's priorities and comparative advantages. However, comprehensive approaches to measure and monitor bioeconomy progress are frequently lacking. Most countries only measure the contribution to gross domestic product (GDP), turnover, and employment of the sectors included in their bioeconomy definition, which may provide an incomplete picture. In addition, this study identifies the mismatch between the targets and measurement methods, as the environmental and social impacts of bioeconomy are often foreseen, but not measured. It is concluded that existing global efforts towards sustainable bioeconomy monitoring can be strengthened and leveraged to measure progress towards sustainable goals.
\end{abstract}

Keywords: bioeconomy; bio-based products; GDP; policy measures; sustainability assessment; sustainable development

\section{Introduction and Background}

Modern economies rely on resources which are finite in nature. On top of their long-term unsustainability, utilization of fossil-fuel resources and unsustainable consumption of derived products also pose risks to societies and the environment due to their negative impacts such as climate change and ecosystem degradation [1,2]. Nevertheless, advancements in industrial biotechnology have enabled the derivation of materials, chemicals, and energy from renewable biomass, which could provide substitutes for fossil-based and finite resources [3]. This substitution potential forms the core of a still-evolving bioeconomy concept.

The literature on bioeconomy vision has been evolving in parallel with the concept and has been clustered under three major perspectives: (1) the biotechnology vision, which emphasizes innovations and utilization of biotechnology at commercial scales; (2) the bioresource vision, which emphasizes the improvement of value chains on upstream biomass production; (3) and the bioecology vision, 
which emphasizes the positive impacts of energy and resource optimization on ecosystem health [4]. These perspectives underline the potential of bioeconomy in the opportunities it offers, such as low-carbon economic growth, preservation of natural resources, restoration of environmental and ecosystem health, and welfare of rural communities.

Due to its promising potential in addressing these global challenges, bioeconomy has been directly or indirectly included in policy agendas worldwide $[5,6]$. Country objectives and bioeconomy priorities encompass economic growth, employment, energy security, food security, fossil-fuel reduction, mitigation and adaptation to climate change, and rural development [7]. With respect to their potentials in deploying the bioeconomy vision, countries have different opportunities, which might also affect their policies. Countries can be classified as countries with: (1) an abundance of renewable biological resources, but a lack of downstream processing industries; (2) both high feedstock potential and advanced processing industries; and (3) low feedstock potential but advanced processing industries [8]. These variations in potential also create differences in countries' objectives for adopting a bioeconomy strategy and in the evaluation of success towards their achievement.

Measuring bioeconomy contribution to countries' overall economy can be an important indicator of development. No internationally agreed methodology exists today to measure progress in attaining the ambitions and targets set by bioeconomy policies and strategies. Moreover, given the differences among countries' constraints, opportunities, and priorities, the development of a uniform way to assess the contribution of bioeconomy to the national economy is challenging. In addition, incomprehensive measurement processes might lead to the omission of potential negative impacts of bioeconomy. This lack of a coherent methodology could also create confusion when trying to compare the importance of bioeconomy within and across countries. One first step towards a globally recognized methodology could be to assess the current efforts of individual countries to define bioeconomy and the frameworks for measuring, monitoring, and reporting its contribution. In fact, regional efforts for the harmonization of the measurement of bioeconomy exist, for example, in the European Union (EU). A European Commission (EC) strategy for the bioeconomy was launched in 2012, and the EC Joint Research Centre (JRC) has been assigned to monitor jobs and turnover in the EU bioeconomy for all the member states and sectors. However, for a global methodology, a more geographically balanced analysis would be useful.

Typical economic models that can be adopted to measure the bioeconomy contribution to a country's economy include the value added/GDP approach, the input-output (I-O) and social accounting matrix (SAM) analysis, the computable general equilibrium (CGE) model, the partial equilibrium (PE) model, and other economic models and tools [9]. However, these approaches do not systematically consider environmental and social aspects. In fact, the objective of this study is to analyze how the contribution of bioeconomy is measured in the overall national economy, using information from a geographically representative list of countries (Argentina, Germany, Malaysia, the Netherlands, South Africa, and the United States). Furthermore, the alignment of the country objectives and the parameters measured have been analyzed in order to assess whether social and environmental impacts of bioeconomy were captured through selected measurement, monitoring, and reporting frameworks adopted by the countries.

Within the scope of this study, the bioeconomy has been defined as "the knowledge-based production and utilization of biological resources, biological processes, and principles to sustainably provide goods and services across all economic sectors" [7]. It involves three elements: (1) the use of renewable biomass and efficient bioprocesses to achieve sustainable production; (2) the use of enabling and converging technologies, including biotechnology; (3) and integration across applications such as agriculture, health, and industry. In accordance with the Food and Agriculture Organization of the United Nations (FAO) development [7], the term "bio-based economy" excluded food and feed production. Instead, it was used to take the production of nonfood goods into consideration, i.e., bio-based materials, chemicals, and pharmaceuticals; pulp and paper; construction materials; textiles; and bioenergy. "Bio-based industry" refers to the industrial production of all possible 
bio-based goods. The strategies related to bioeconomy, bio-based economy, and bio-industries were all considered as "bioeconomy strategies". This assessment has been built upon previous and ongoing efforts to foster global bioeconomy. In this respect, the countries to be investigated have been selected among the members of the FAO International Sustainable Bioeconomy Working Group (ISBWG), which includes 23 members as of March 2018: 11 countries (Argentina, Brazil, China, Germany, Italy, Kazakhstan, Malaysia, the Netherlands, South Africa, Uruguay, and the United States), the German Bioeconomy Council, the European Union (EU) Commission, Organisation for Economic Co-operation and Development (OECD), International Center for Tropical Agriculture (CIAT), Stockholm Environment Institute (SEI), United Nations Environment Programme (UNEP), World Wide Fund (WWF), the Nordic Council of Ministries, the EU Bio-Based Industries Consortium, Wageningen University, the World Business Council for Development (WBCSD), and FAO.

The countries selected for the study are located in five continents, differ in terms of levels of economic development, and have different bioeconomy strategies and priorities. For instance, some countries have little land availability but advanced technologies, while others prioritize farmers and rural development and have greater land availability. For all the countries, the study reviews bioeconomy objectives and priorities as well as measurement, monitoring, and reporting frameworks. From the sample countries and a review of existing literature, a pathway towards a sustainable bioeconomy monitoring is then proposed.

\section{Approach and Methods}

The study was based on desk research of policy documents, strategies, and statements on the bioeconomy and its measurement for a selected number of countries (Argentina, Germany, Malaysia, the Netherlands, South Africa, and the United States). Because of their demonstrated interest in developing a global bioeconomy framework, the selection process was primarily performed among the ISBWG member countries, following the selection process illustrated in Figure 1. The structural organization of the analysis included an evaluation of the available information in terms of: (1) how countries define bioeconomy; (2) which are the objectives and/or priorities of their strategy; (3) and the methodology they use to measure, monitor, and report the contribution of bioeconomy to their economy or objectives (Figure 1).

The relevant information for the analysis was gathered from official bioeconomy strategies and documents, upon availability. When a government official document on how to measure the contribution of bioeconomy to the total economy was not available, commissioned studies, studies from research institutes, and/or non-profit organizations were used instead. Table 1 summarizes the sources of information and documents analyzed in order to understand the definition of bioeconomy, the objectives/priorities of the bioeconomy strategy, and the measurement, monitoring, and reporting frameworks established by each selected country. Whenever possible, the information was complemented and validated by a survey, which was distributed to government representatives (Table 2). The survey included the same questions to which answers were sought while scanning through the written materials. 
Table 1. Sources of information for the selected countries.

\begin{tabular}{|c|c|c|c|}
\hline & Bioeconomy Definition and Strategy & Objectives/Priorities of the Strategy & $\begin{array}{l}\text { Measurement, Monitoring and } \\
\text { Reporting Framework }\end{array}$ \\
\hline Argentina & Ministry of Agroindustry (MINAGRO) [10] & MINAGRO [10] & Bolsa de Cereales [11] \\
\hline Germany & $\begin{array}{l}\text { Federal Ministry of Food and Agriculture (BMEL), } \\
\text { and the Federal Ministry for Education and } \\
\text { Research (BMBF) [12] }\end{array}$ & National Policy Strategy on Bioeconomy [13] & $\begin{array}{l}\text { A comprehensive and system monitoring approach } \\
\text { to measure the contribution of German BE to the } \\
\text { overall economy is currently under development }\end{array}$ \\
\hline Malaysia & $\begin{array}{l}\text { National Biotechnology Policy (NBP); } \\
\text { the BioNexus Status (BNX); the Bioeconomy } \\
\text { Transformation Programme (BTP); } \\
\text { the Bioeconomy Community Development } \\
\text { Programme (BCDP); National Biomass Strategy } \\
2020 \text { ("NBS 2020") [14] }\end{array}$ & BTP and BCDP [14] & MOSTI and Bioeconomy Corporation $([14,15])$ \\
\hline The Netherlands & $\begin{array}{l}\text { Netherlands Enterprise Agency (RVO) [16]; } \\
\text { CE Delft [17]; Agency Ministry of Economic } \\
\text { Affairs (NOST) [18]; NNFCC [19] }\end{array}$ & NOST [18]; NNFCC [19] & $\begin{array}{l}\text { Bio-based economy protocol monitor [16]; } \\
\text { NOVA Institute [20]; CE Delft [17]; EC Bioeconomy } \\
\text { Knowledge Centre [21] }\end{array}$ \\
\hline South Africa & $\begin{array}{l}\text { Public Understanding of Biotechnology [22]; } \\
\text { National Biotechnology Strategy [23] }\end{array}$ & National Biotechnology Strategy [23] & $\begin{array}{l}\text { National Biotechnology Strategy [23]; ongoing study } \\
\text { to establish a framework to develop indicators to } \\
\text { measure the growth of the BE in South Africa [24] }\end{array}$ \\
\hline
\end{tabular}




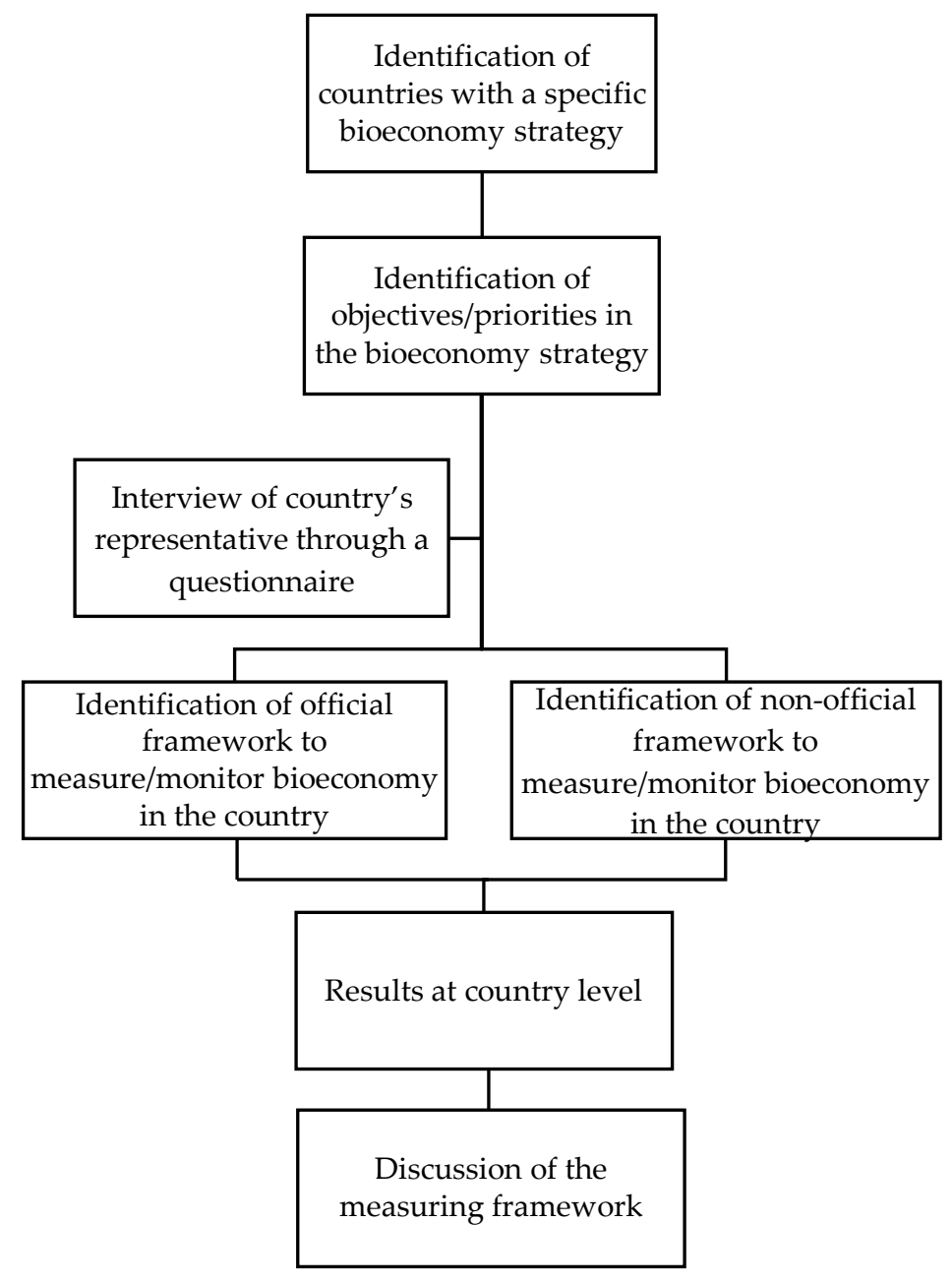

Figure 1. Selection process and structural organization of the analysis.

Information collected through desk research and surveys has been used for the analysis and has been complemented with an extensive literature review of objectives and priorities of bioeconomy strategies in other low-, middle-, and high-income countries (outside the study focus) in order to improve the quality of the discussion. For this purpose, the survey was distributed to all ISBWG members (not only to those in the study focus).

Table 2. Questionnaire submitted to ISBWG members on assessing bioeconomy contribution to countries' economy.

\begin{tabular}{l}
\hline Bioeconomy Definition \\
\hline How does your Country define bioeconomy? \\
\hline Which sectors are included into your bioeconomy strategy? \\
\hline (e.g., Agriculture; Automotive and mechanical engineering; Chemistry (incl. bioplastics); Biofuels/bioenergy; \\
Biorefining; Construction/Building industry; Consumer goods such as cosmetics and cleaning products; Feed; \\
Fisheries; Food and Beverage industry; Forestry; Health; Knowledge/Innovation; Mining; Pharmaceuticals \\
industry; Pulp and paper; Textiles) \\
\hline Objectives/Priorities \\
\hline $\begin{array}{l}\text { Which are the objectives / priorities of your country strategy (e.g., food security, energy security, fossil fuel } \\
\text { reduction, rural development, economic growth, employment, mitigation and adaptation to climate } \\
\text { change, etc.)? }\end{array}$
\end{tabular}


Table 2. Cont.

\begin{tabular}{l}
\hline Measurement, Monitoring and Reporting Framework \\
\hline Does the country strategy include criteria to measure the contribution of bioeconomy to the overall economy? \\
If yes, which ones? \\
\hline Which approach does your country use to measure bioeconomy contribution? (e.g., GDP approach; \\
Input-Output matrix; Computable General Equilibrium (CGE) Model; Partial Equilibrium (PE) Model) \\
\hline Does your country measure the impact of bioeconomy on the following areas? (Turnover/sales; Value added; \\
Job creation; Market development; Investments; Intellectual property; R\&D spending; Trade balance; Poverty \\
alleviation; Food security and sustainable agriculture; Health and well-being; Education; Gender equality; \\
Availability and sustainable management of water; Access to affordable, reliable, sustainable and modern \\
energy; Inclusive and sustainable industrialization and innovation; Inequality and inclusiveness; Inclusive, \\
safe, resilient and sustainable cities; Ensure sustainable consumption and production patterns; Climate change; \\
Oceans, seas and marine resources; Terrestrial ecosystems, forests, land degradation and biodiversity) \\
\hline Are indicators to measure bioeconomy contribution defined? If so, which ones? \\
\hline Short Discussion \\
\hline Which is, in our opinion, the main limitation of your country approach to measure bioeconomy contribution? \\
\hline
\end{tabular}

\section{Analysis and Results}

\subsection{Bioeconomy Definitions and Strategies}

It is observed that the sectors and subsectors considered in bioeconomy were different among the analyzed countries, which is a reflection of the differences in their priorities and strategies. Table 3 summarizes the various sectors included in the bioeconomy definition by the six analyzed countries, and the sectors taken into account for the quantification of bioeconomy contribution to overall national economy. The first important point is the variation in the definition of bioeconomy. For instance, the Netherlands has a focus on bio-based economy, excluding the agriculture and food sectors [17] and still has not agreed on a which sectors are included in the bioeconomy [31]. The United States aims to analyze the bioeconomy, but USDA shows results limited to bio-based products industries, which exclude the energy, food, feed, livestock, and pharmaceutical industries.

Table 3. Sectors included into BE strategy and monitoring in the selected countries.

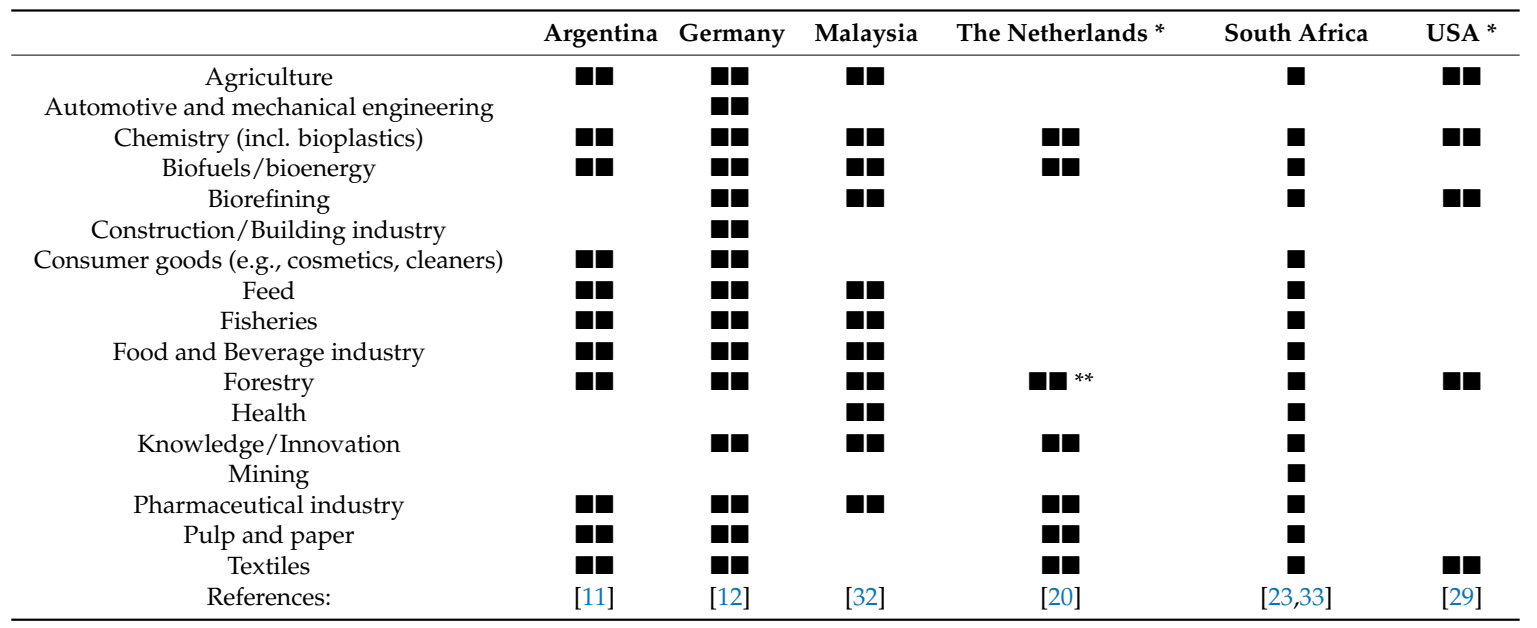

* The monitoring system analysis for the Netherlands refer to bio-based economy and the results for the United States refer to bio-based products industries. ${ }^{* *}$ Only forest-based industry. Legend: $\mathbf{\square}$ : included in bioeconomy strategy, $\mathbf{\square}$ : included in the bioeconomy strategy and monitored or measured.

According to Argentina, the bioeconomy includes agriculture, forestry, fishing, food production, and pulp and paper production, as well as parts of textile, chemical, energy, and biotechnological 
industries (medical and pharmaceutical industry). The German bioeconomy includes agriculture, forestry, fishing, manufacturing, and trading of bio-based products. Similarly, the Malaysian bioeconomy includes agriculture, forestry, fisheries, food, feed, healthcare wellness products, chemicals, and renewable energy. South Africa's bioeconomy strategy focuses on agriculture, industrial, and environmental bio-innovation and health but has yet to develop metrics to monitor performance.

It is worthwhile to note that the decision on which sectors to include is also relevant when trying to measure the bioeconomy's contribution to countries' economies in terms of GDP and value added, since the calculations would only take the included sectors into account [34].

\subsection{Bioeconomy Objectives and Priorities}

The sectors included in the bioeconomy strategy often reflect the priorities identified by the country and comparative advantages linked, for instance, to endowment in biomass resources, historical economic specialization, labor productivity, and past investments in R\&D [7,11,35-39].

For instance, in Argentina, bioeconomy is seen as a tool for sustainable development in the country. It is recognized as a positive alternative for the generation of new behaviors and sources of employment to face the double challenge of climate change and the continued need for economic progress indispensable for poverty reduction [10].

In Germany, the National Policy Strategy on Bioeconomy's priorities for advancing towards a knowledge-based bioeconomy are: the development of a secure supply of high-quality food; the transition from a fossil-based economy to an economy that is increasingly efficient in terms of raw materials and based on renewable resources; the supply of renewable resources; the sustainable use of renewable resources while conserving biodiversity and soil fertility; protection of the climate; the strengthening of Germany's innovative power and its international competitiveness in business and research; securing and creating employment and added value, particularly in rural areas; and sustainable consumption [13].

In Malaysia, bioeconomy is seen as a key contributor to economic growth, which can provide benefits to the society via breakthroughs in agricultural productivity, innovations in healthcare, and the adoption of sustainable industrial processes [15].

The objectives of the South African bioeconomy strategy are to make the country more competitive internationally (especially in the industrial and agriculture sectors), to create more sustainable jobs, to enhance food security, and to create a greener economy as the country shifts towards a low-carbon economy [23]. In particular, the strategic economic sectors identified are (i) agriculture, (ii) health, and (iii) industry and environment.

In the Netherlands, the bioeconomy strategy encourages knowledge development and innovation in nine top sectors: agriculture and food, water, chemicals, energy, life sciences and health, horticulture and propagation materials (seed stock), logistics, high-tech systems, and materials and creative industries [18]. However, the Dutch government focuses more often on the bio-based economy, defined as "economic activity based on biomass, with the exception of human food and feed", with the condition that it is based on recently captured carbon [40]. The most important drivers behind the adoption of a bio-based economy strategy were: striving for more sustainability (reduction of $\mathrm{CO}_{2}$ emissions, circular economy); the awareness of the finite nature of fossil fuels; and the economic opportunities offered to Dutch businesses through the use of renewable biological resources and residues [18].

In the United States, the five strategic objectives introduced by the National Bioeconomy Blueprint aimed to generate economic growth and address societal needs. Some of these strategies include supporting R\&D investments, facilitating the transition of bio-inventions from research lab to market, developing and reforming regulations, updating training programs, and aligning academic institution incentives with student training for national workforce needs. They also include identifying and supporting opportunities for the development of public-private partnerships and precompetitive collaborations in their objectives [25]. 


\subsection{Measurement, Monitoring, and Reporting Frameworks}

The countries selected for the study use various approaches for measuring the bioeconomy contribution to their economy and in attaining the objectives.

In the case of Argentina, a standard approach was adopted to measure the contributions (gross production value and value added) of bio-based products to the national GDP, referencing the general principles of the System of National Accounts (SNA) for the calculation of GDP and internationally comparable satellite accounts (e.g., for education, capital, productivity, and environment). A paper by an intermediate service provider Bolsa de Cereales [11] designs a general methodology for the criteria, procedures, and databases to be used in the measurement of bioeconomy and its contribution to GDP. The sectors included in the calculation of the bioeconomy's contribution to the GDP are "agriculture, forestry, fishing, food production, and pulp and paper production, as well as [bio-based] fractions of textile and chemical industry, and energy and biotechnological industries (health and pharmaceutical industry)" [11], which are in alignment with the sector included in the country's bioeconomy strategy. However, the study from Bolsa de Cereales considers only the economic variables, without taking into account the social and environmental aspects of the bioeconomy. For instance, the study does not report on regional and territorial development, employment, food security, energy security, sustainability, or climate change mitigation and adaptation, which are among the objectives of the bioeconomy vision. Not addressing these issues would not only give an incomplete picture of the achievement of these objectives, but it also might pose the risk of overlooking the potential negative impacts of bioeconomy on these dimensions.

In Germany, most of the areas contributing to bioeconomy are monitored by traditional statistical accounts [12]. However, in most of the cases, methodologies for data collection and assessment are not streamlined to assess the impact of the bioeconomy. This leads to sparse information on impacts, along with data gaps, uncertainties, lack of comparability of results, and the potential double counting of impacts. A comprehensive and system monitoring approach to measure the contribution of German bioeconomy to the overall economy is currently under development by a joint interministerial undertaking, consisting of three main projects: the monitoring of biomass flows, the Systemic Monitoring and Modelling of the Bioeconomy (SYMOBIO), and the identification of economic key performance indicators to monitor the bioeconomy [41].

Malaysia has developed a Bioeconomy Contribution Index (BCI) to measure the contribution of bioeconomy to the overall economy, which is a combination of five components/parameters: bioeconomy value added, bio-based exports, bioeconomy investments, bioeconomy employment, and productivity performance [14]. The $\mathrm{BCI}$ is a comparative tool designed to provide a holistic view, encompassing multiple aspects of the bioeconomy, and it is used to identify trends, patterns, and synergies within the industry. The index compares the performance of each specific component for a selected year against the adjusted (i.e., accounting for changes in variables such as inflation rates and import-export values) expected base performance (in the base year 2005), determined by a dynamic computable general equilibrium (DCGE) model [15]. The share of bioeconomy contribution to national development is estimated from the SAM, assuming that Malaysia is a price taker country [42]. Until now, the BCI has been primarily measuring revenues and economic flows, but it could be further improved to consider broader socioeconomic or environmental aspects. For instance, the BCI could incorporate social measures (e.g., poverty reduction and income inequality in the bioeconomy industry), and environmental measures (e.g., $\mathrm{CO}_{2}$ emissions and level of local biodiversity), in order to evaluate whether the bioeconomy poses a risk or contributes to sustainability in all dimensions [42].

Since the Netherlands lacks a clear protocol on defining the boundaries of the bioeconomy, until now their focus has been on the bio-based economy (BBE). In 2013, the Netherlands established a BBE monitoring protocol to quantify its size and to monitor its development over time in order to make trends visible and comparable with developments abroad [16]. The protocol defines the system boundaries, the units to express the size of BBE, and utilization of available data or their collection, if missing. The protocol is built on existing statistical data on production and consumption, 
prevents double counting of bio-based raw materials, and accounts for raw material flows monitoring. However, it raises some problems linked to classification of business sectors, classification of product groups, and timely acquisition of data [16].

The South African bioeconomy strategy [23] contains some indicators to monitor progress in the bioeconomy in comparison with other high- and middle-income countries, broadly divided into "knowledge and skills" indicators (full-time equivalent researchers, scientific publications, and bioeconomy-related publications) and "financial support" indicators (gross domestic expenditure on R\&D as percentage of GDP and funding and governmental support). Moreover, the methodology provides 18 output indicators (related to industry, market, knowledge transmission and application, and knowledge base and human resources) to be used to track and monitor the bioeconomy strategy [23]. However, systematic metrics to measure and monitor South Africa's bioeconomy have not yet been implemented. Moreover, most indicators in the strategy are derived from the measurement of a knowledge-based economy or biotechnology innovation policies. Therefore, they do not cover social and environmental issues, which would make it difficult to determine negative impacts, if they exist, on these aspects. Ongoing efforts are anticipated to result in detailed implementation plans and value propositions for specific sectors and initiatives that will help refine targets [33].

The USDA report [29] examines and quantifies the effect of the bio-based products industry from an economics and jobs perspective at the state level. It was preceded by a report analyzing the effect at national level [28] and by another report [43] which provides a snapshot of available information on the bioeconomy in the country and a platform upon which to build future efforts to measure the bioeconomy. The report adopts a three-pronged approach to gather information: interviews of representatives of government, industry, and trade associations involved in the bio-based products; data collection from government agencies and published literature on the bio-based products industry; and economic modelling. Despite being intended as a platform for understanding and tracking the progress of the bioeconomy in the United States, the USDA 2016 report does not provide a complete picture of the bioeconomy, as it does not report on the bioenergy sector, which is included in the US strategy. Instead, it only focuses on seven major sectors chosen to represent the bio-based industry's contribution to the US economy (agriculture and forestry, biorefining, bio-based chemicals, enzymes, bioplastic bottles and packaging, and forest products and textiles). In 2017, the Department of Energy (DOE) also provided some figures about the size of the bioeconomy [30], building up the Billion Ton Bioeconomy Vision, but without a systematic measurement approach. The DOE estimates are taken from a paper considering direct employment and revenues from biomass resources fed into a number of end-uses and products including heat and power generation, bio-based chemicals and products (including wood pellets), and biofuels and coproducts [44].

\subsection{Limitations in Data Availability and Statistical Approaches}

Most of the analyzed countries currently measure the contribution of bioeconomy to their GDP and other economic variables only. This economic approach, however, has some limitations in reflecting the contribution in the economic sphere, above all because no standard methodology has been established to enable international comparison of bioeconomy contribution to GDP. Additionally, as mentioned above, products and activities comprised within the bioeconomy greatly vary according to country's priorities and comparative advantages.

The most common classifiers of economic activity, trade and products at the international level (International Standard Industrial Classification (ISIC), North American Industry Classification System (NAICS), Classification of Economic Activities in the European Community (NACE), Nomenclature for External Trade (NET), and Classifier per Category (CPC)) are not compatible with the complexity of the bioeconomy $[11,37]$ since they are not appropriate for the heterogeneous nature and variety of bio-based products. ISIC, NACE, and NAICS group production units according to the similarity of their productive processes, technology, inputs, and equipment. Their classification criteria make no distinction between bio- or nonbio-inputs [11]. Even the System of National Accounts (SNA 08) 
from the United Nations, which provides recommendations for measuring the national production, wellbeing, and other economic issues in an internationally comparable way, does not allow for the measurement of the bioeconomy [11]. Classifiers based on traditional industrial activities are not compatible with the bio-based industry. This can lead to under or overestimation of the size of the bioeconomy.

The high number of bio-based products and their heterogeneity make it very difficult to provide a full quantitative picture of the status and evolution of the bioeconomy [45]. Often, data on the bioeconomy are retrieved from surveys of the bio-based industry $[11,27,46]$. These surveys represent an important first step for a systematic approach to quantify the bioeconomy. However, they face difficulties in assembling the requested data and suffer from incomplete response rate [46]. These limitations are even more relevant in low- and middle-income countries, where statistical systems are not well developed. Under these circumstances, the surveys may not be updated and/or may include limited and biased samples (as shown for instance by the Argentinian and South Africa analyses, where the last company surveys were taken in 2003 [47]). Digitalization efforts as the ones undertaken in Malaysia to improve data collection can play an important role in the measurement and monitoring of bioeconomy.

\section{Discussion}

\subsection{The Need for Defining the Bioeconomy Boundaries at National, Regional, and Global Levels}

Due to the lack of a homogenous definition of bioeconomy and its sectors, a common ground which enables comparing the contribution of bioeconomy among countries is missing. Also, at national level, the definition of the bioeconomy boundaries is sometimes unclear. For instance, in the case of the Netherlands, the estimated impacts of bioeconomy were different among the official studies analyzed due to the variety of methodologies and input data $[17,20,21]$. Also in the United States, most of the sectors considered by the DOE to estimate the size of bioeconomy were excluded from the USDA 2016 report, leading to different estimates of the bioeconomy impacts in the country $[29,30]$. For this reason, the efforts of the US Biomass R\&D Board to coordinate programs within and among departments and agencies of the federal government towards a single, harmonized bioeconomy vision should ideally produce a single comprehensive approach able to monitor and measure all the sectors included in the vision in a coherent way.

When the countries do not have a holistic bioeconomy strategy, they tend to adopt a fragmented approach by separately considering the different uses of biomass in each sector (e.g., agriculture, forestry, energy, and transport). This approach to governing the bioeconomy leads to different policies for different uses of biomass, different incentives for investment, and different regulations for the areas from which feedstocks are sourced [48]. In these countries, the efforts should aim at integrated approaches across different levels, sectors, landscapes, and end-uses in order to avoid boom and bust policies as it happened for first-generation biofuels in the EU and elsewhere.

Nevertheless, some regional efforts to harmonize the measurement of the bioeconomy's economic significance exist. For instance, since the launch of the European Commission (EC) strategy for the bioeconomy in 2012, the EC Joint Research Centre (JRC) is monitoring jobs and turnover in the European Union bioeconomy for all the member states and sectors [49]. More specifically, the EC Bioeconomy Knowledge Centre shows turnover, employment, and location quotient (i.e., the share of employment in the bioeconomy in a member state divided by the EU employment share in the bioeconomy) [49]. In order to enable achievement of the full potential of the bioeconomy, global guidelines on the measurement and regulation of the value chains could be beneficial [8]. FAO has been already coordinating the global efforts towards the development of international Bioeconomy Sustainability Guidelines. These could be used by the countries to measure sustainability aspects of their bioeconomy strategy and monitor the achievement of economic, social, and environmental targets and priorities. 


\subsection{Bioeconomy as a Means to Achieve Sustainable Development Goals}

The bioeconomy has already been adopted by a significant number of countries as a new vision of development to decouple the economy from the fossil-fuel dependence and as a valid path towards the achievement of the Sustainable Development Goals (SDGs) and the commitments under the Paris Climate Agreement. For instance, efficient and sustainable natural resource management is directly tied to at least 12 of the 17 SDGs and can cut greenhouse gas (GHG) emissions by $60 \%$ by 2050 [50]. In addition, for lower-income countries, better management of natural resources is often a key component of poverty eradication, climate-change mitigation, and resilient economic growth [51].

In low- and middle-income countries with available biomass resources and/or well-developed primary sectors, a sustainable bioeconomy could unlock new opportunities for economic development and industrialization and support economic and social objectives, such as reducing unemployment and expanding access to energy. For instance, in Argentina (and similarly in other Latin American countries with high feedstock availability), the increase in the value added to agricultural production can create employment and improve the competitiveness of export-oriented sectors. The agriculture sector of the region has the potential to generate productivity gains, which could result in significant improvements in countries' inclusion in international trade [11]. Improvement in agricultural productivity can also play an important role in building resilience while increasing yields for farmers [35]. Countries with a low labor-productivity level in the bioeconomy sectors but abundant primary production and a sound manufacturing base could add value through bio-based methods of production [37].

The agriculture sector is also a key component of the bioeconomy strategy for middle-income countries such as Malaysia and South Africa. In Malaysia, the performance of the palm oil sector seems to somewhat determine the overall direction of Malaysian bioeconomy development [36]. In South Africa, enabling job creation through the expansion and intensification of sustainable agricultural production and processing is part of the three strategic objectives of the bioeconomy.

In the United States, with both high feedstock potential and advanced industries, the bioeconomy is based both on the expansion of biomass and on 'bio-inventions' [25,52]. However, a bioeconomy vision based on the expansion of biomass can face challenges, such as the reliable availability of raw materials due to the increased climate and severe weather impacts, water availability, and stability of the markets [43].

In contrast, some high-income countries such as the Netherlands have excluded the agriculture and food sector from their bio-based economy strategy. The main reasons for this is the limited domestic supply of ecologically sustainable biomass, which concerns several other EU countries as well. Estimates suggest that, for the EU, the sustainable biomass supply will be enough to meet about 10-20\% of the final energy and feedstock consumption in 2030 [39]. Considering that land use is the most critical issue in sustainable biomass production, countries with limited land availability face relevant constraints. In countries with limited biomass availability, such as some Western European countries, the bioeconomy strategies focus more on biochemistry and bio-pharmacy benefiting from long-standing experience and R\&D investments [49]. In countries focusing on high value-added bioeconomy sectors, the bioeconomy can generate higher turnover compared to the employment generated, whereas the less value-added sectors of the bioeconomy (mainly primary biomass production in agriculture, forestry, and fisheries) typically generate more employment.

Technical innovation and new business models associated with the bioeconomy should also potentially aim at decoupling economic growth from resource use in countries with available resources [51]. A sustainable bioeconomy would not foster depletion of resources, degradation of the environment, loss of biodiversity, and social injustice. As Germany recognizes in its bioeconomy strategy, the structural transition towards a bio-based economy can only be successful if it secures the supply of food, it protects the environment, the climate, and biodiversity, and it supports the development-policy objectives in developing countries and emerging economies [13]. 


\subsection{Linking Goals and Measurement Frameworks}

If a bioeconomy strategy aims to contribute to sustainable development and to environmental and social objectives (e.g., employment, food security, energy security, and mitigation and adaptation to climate change [10]), these should be clearly included in the strategy objectives and should be measurable (by means of quantitative, qualitative, or as aggregate indicators). Environmental and sustainability components in bioeconomy development approaches should be closely connected with supply and production of bio-resources, as well as with consumption patterns. In fact, the core of transformational strategies is not limited to the technological aspects but includes behavior change and institutional innovations for enabling settings and long-term incentives, both at the company and of international policy levels [38].

This study shows that a means to monitor progress in reaching the targets set in the bioeconomy policies and strategies is lacking in many countries, and the difficulty of measuring it can be a consequence of the lack of a clear definition of the bioeconomy concept and of concrete and measurable objectives. In fact, strategies often show nonmeasurable objectives and qualitative targets. In the case of South Africa and the United States, for example, the suggested output indicators of critical factors to monitor bioeconomy strategy [23] and the bio-based economy indicators and composite indicators [27] have not been measured in the practice yet due to the lack of sufficient data.

Most countries monitor bioeconomy progress just with economic values and shares of GDP, while other aspects of sustainability and resource availability are addressed only to a limited extent [53]. The GDP is a parameter which certainly gives information on the bioeconomy contribution to the economy. However, it is not ideal due to the inadequacy of the standard industrial classification systems to systematically monitor bio-based production, the lack of systematic data, and the often scattered information collected at national level. In addition, GDP is being increasingly criticized as an inappropriate indicator to measure sustainable development since it includes activities considered detrimental to humans and the environment and does not take into consideration social aspects that define human wellbeing nor the environmental aspects (which are all important information to assess the real contribution to the overall economy). Moreover, the GDP does not include transfer payments, such as subsidies for fossil fuels [54].

In addition to GDP, other economic indicators often used to measure bioeconomy are: turnover (revenue from sales); employment; resource use (crops, wood, waste, land, capital, etc.); primary production of biomass in the country (agriculture, forestry, residues, fisheries, and waste); import of biomass to the country; global land use for biomass based consumption in the country; production of bio-based products; price of biomass and bio-based products; consumption of bioeconomy products; and trade flows $[18,27,34,49]$. Further indicators focus on the drivers of innovation, such as investments and spending in R\&D or intellectual property. However, it can be difficult to capture the impacts of a new innovation due to a time lag between investments and outcomes. These types of indicators could be used to compare country performance in the development of a bioeconomy (e.g., which countries have a bioeconomy strategy or have dedicated R\&D funds).

Some countries currently measure only the effect of bio-based products on the GDP, although bioeconomy related services could be included in the measurement of the bioeconomy. For instance, Finland includes nature tourism, hunting, and fishing as bioeconomy services in its bioeconomy strategy [55]. Also, the Malaysian BCI currently measures primarily revenues and economic flows, but it could be improved to take bio-services into account as well as broader socioeconomic or environmental aspects. For instance, the BCI could incorporate measures of poverty reduction or income inequality in the bioeconomy industry, it could account for $\mathrm{CO}_{2}$ emissions, or level of local biodiversity [42].

Nevertheless, some efforts to develop measurable social and environmental indicators to monitor the bioeconomy exist. For instance, Italy has developed a set of sustainability indicators with measurable impacts on food security, natural resources sustainability, dependence on nonrenewable resources, and climate change, in addition to economic growth [56]. These indicators on sustainability 
dimension are based on the results of Systems Analysis Tools Framework for the EU Bio-Based Economy Strategy (Sat-BBE) consortium [34]. In order to measure the environmental impact of the bioeconomy, the EC JRC has developed and integrated modelling framework (IMF) to implement the consequential life cycle assessment (C-LCA). This framework identifies the consequences that a decision in the foreground system has for other processes and systems of the economy, both in the analyzed background system and on other systems outside the boundaries, and allows policy impact assessment once it is fully implemented [46]. However, data gaps still need to be filled and concepts and methodology, including the IMF for the environmental impact assessment, need to be further developed and implemented.

Other environmental assessment and environmental management techniques include carbon footprinting, eco-audit, environmental and social impact assessment, and strategic environmental assessment [57]. An ongoing project, MontBioEco (from the Natural Resources Institute Finland (Luke), the Standing Committee on Agricultural Research (SCAR) Bioeconomy Strategic Working group (BSW), and CASA Ministry of Agriculture and Forestry Finland (MMM)) is developing a synthesis on bioeconomy monitoring systems in the EU Member States, including indicators and subindicators. The analysis has currently developed 22 indicators and 146 subindicators around 5 main objectives: creating jobs and maintaining competitiveness; reducing dependence on nonrenewable resources; mitigating and adapting to climate change; and ensuring food security and managing natural resources sustainably [58]. This assessment includes the development of measurable indicators and subindicators that go beyond economic monitoring.

In countries with an existing bioenergy or biofuels strategy, efforts towards monitoring the sustainability of bioeconomy can be linked with the previous efforts on biofuels, biomass, and bioenergy. Moreover, several standard, certification, and labelling initiatives already set some indications both on the "quality" of the bio-products and on their sustainability. For instance, the USDA has developed a Certified Biobased Product label which certifies the carbon content of a number of bio-products. Many existing bio-product certifications and standards give indications for monitoring environmental and social sustainability.

A further step in monitoring bioeconomy should ideally include the measurement of taxation and regulatory support. For instance, the Dutch RVO estimates also the investment in BBE R\&D through tax credits and fiscal exemptions [20].

Other national studies are analyzing existing policy to assess how public finance, regulations, and capacity building can enable growth of the bioeconomy (see for instance [59] for Thailand).

Bioeconomy is also an opportunity for young people and next generations, and it is often linked to improving science, technology, engineering, and mathematics (STEM) education and training programs to meet the workforce needs. For instance, these aspects come as a key priority in the US bioeconomy strategy and the Finnish bioeconomy strategy, for which developing the bioeconomy competence base by upgrading education, training, and research is a key objective [55]. Also, the South African bioeconomy strategy and the indicators suggested in the strategy, being driven by the Department of Science and Technology, have an "innovation" bias. In fact, most indicators in the strategy are related to science, technology, and innovation, as they are derived from the measurement of a knowledge-based economy or biotechnology innovation policies [23].

Finally, in Argentina, Malaysia, and South Africa, one of the bioeconomy objectives is to strengthen infrastructure to support economic growth and increase access to national and international markets $[10,15,23]$. Therefore, this aspect should ideally be included in a pathway towards a sustainable bioeconomy monitoring including socioeconomic and environmental impacts. Other aspects, such as the inclusion of gender (not mentioned as priority in any of the strategy analyzed), may be included in the measurement framework, in order to reflect country's priorities and strategy. Consideration of social and environmental aspects would not only minimize the risks associated with bioeconomy transition but would also enable evaluation of the real picture of the bioeconomy impacts. Similarly, in countries heavily depending on the import of biomass, a broader perspective on the evaluation 
of bioeconomy would enable internalization of the potential negative impacts on societies and the environment at a global scale [60].

\section{Conclusions}

This study underlines the lack of a homogenous definition of bioeconomy across the countries analyzed, which does not allow any straightforward comparison of the relevance of bioeconomy in the different economies.

The sectors comprised mostly reflect priorities identified by the country and comparative advantages linked, for instance, to availability of natural resources, traditional industries, labor productivity, and past investments in R\&D. For instance, the agri-food sector is identified as a priority for Argentina, Malaysia, and South Africa, while the Netherlands and the Unites States focus more on nonfood sectors.

Most countries measure bioeconomy progress just with economic values and shares of GDP. On top of the lack of international consensus on which products and activities are comprised within the bioeconomy, the GDP approach has several limitations due to the inadequacy of the standard industrial classification systems to systematically monitor bio-based production, the lack of systematic data, and the often scattered information collected at national level.

Some ongoing efforts aim to harmonize the definition and measurement of bioeconomy, at least across macroregions (such as the EU) will allow development of structured and comparable measurement and monitoring methodology of the trends in bioeconomy, at least for some sets of countries (as the EC already does for few economic indicators).

Usually, bioeconomy strategies also consider intangible aspects, such as institutional set-up, policies, governance, regulations, incentives, and financial instruments, which create an enabling environment for the bioeconomy as well as social and environmental issues. Several countries emphasize the role that bioeconomy plays in their development strategy, which is an important aspect to reflect in their measurement efforts. This could allow monitoring, for instance, progress in meeting the SDGs or environmental targets. In fact, important synergies between countries commitments towards the measurement of SDGs and bioeconomy can be leveraged.

In order to facilitate the measurement and monitoring of bioeconomy at a national level, the governments could enhance and coordinate communication between different domestic agencies and entities and establish protocols for sharing data, formalize bio-based industry measurement standards, develop a comprehensive survey for bio-based industry and commodity usage, and review and revise industry classification systems. Ongoing efforts aim to harmonize the definition and measurement of bioeconomy, at least across macroregions such as the EU. These efforts will allow for the structured and comparable measurement and monitoring of the trends in bioeconomy. These efforts should go hand in hand with the development of relevant and comprehensive guidelines on how to measure the sustainability of the bioeconomy, possibly agreed at an international level. These sets of indicators should also consider the social and environmental dimensions of sustainability, since their omission or exclusion might lead to an overlook of the potential stresses caused by bioeconomy on the social wellbeing of communities and the environmental viability of ecosystems.

Author Contributions: M.G.S.J., S.B. and A.F. conceived and designed the paper; S.B., O.C. and M.G.S.J. conducted scientific literature review and collected the data; S.B. and A.F. analyzed and interpreted the data; S.B. and O.C. wrote the paper; A.F. supervised the work.

Acknowledgments: This study was funded in the framework of FAO project GCP/GLO/724/GER "Towards Sustainable Bioeconomy Guidelines (SBG)" supported by the German Federal Ministry of Food and Agriculture (BMEL). Inputs and comments were provided by several members of the International Sustainable Bioeconomy Working Group (ISBWG). The authors are particularly grateful to Olivier Dubois, FAO Senior Natural Resources Officer; Zurina Che Dir, Bioeconomy Corporation Malaysia; Ben Durham, Department of Science and Technology, South Africa; Mariano Lechardoy, Argentine Ministry of Agroindustry; Marte Mathisen, Nordic Council of Ministers; Elina Nikkola, Finnish Ministry of Agriculture and Forestry; Timothy Ong, Malaysia's National Innovation Agency; Enrico Prezio, European Commission; Tilman Schachtsiek, German Ministry for Agriculture and Food; Cinzia Tonci, Italian Ministry of Economic Development; Jan W.J. van Esch, Dutch Ministry of 
Economic Affairs and Climate Policy; Francis X. Johnson, Rocio A. Diaz-Chavez and Matthew Fielding, Stockholm Environment Institute, for their valuable inputs.

Conflicts of Interest: The authors declare no conflict of interest. The founding sponsors had no role in the design of the study; in the collection, analyses, or interpretation of data; in the writing of the manuscript, and in the decision to publish the results. The views expressed in this report reflect those of the authors and do not necessarily reflect the views of the Food and Agriculture Organization of the United Nations.

\section{References}

1. McCormick, K.; Kautto, N. The Bioeconomy in Europe: An Overview. Sustainability 2013, 5, $2589-2608$. [CrossRef]

2. De Besi, M.; McCormick, K. Towards a bioeconomy in Europe: National, regional and industrial strategies. Sustainability 2015, 7, 10461-10478. [CrossRef]

3. Richardson, B. From a fossil-fuel to a biobased economy: The politics of industrial biotechnology. Environ. Plan. C Gov. Policy 2012, 30, 282-296. [CrossRef]

4. Bugge, M.M.; Hansen, T.; Klitkou, A. What is the bioeconomy? A review of the literature. Sustainability 2016, 8. [CrossRef]

5. German Bioeconomy Council. Bioeconomy Policy (Part II): Synopsis of National Strategies around the World; German Bioeconomy Council: Berlin, Germany, 2015.

6. Viaggi, D. Towards an economics of the bioeconomy: Four years later. Bio-based Appl. Econ. 2016, 5, 101-112. [CrossRef]

7. Food and Agriculture Organization of United Nations (FAO). How Sustainability Is Addressed in Official Bioeconomy Strategies at International, National, and Regional Leveles-An Overview; Food and Agriculture Organization of United Nations: Rome, Italy, 2016; ISBN 978-92-5-109364-1.

8. Axelsson, L.; Franzén, M.; Ostwald, M.; Berndes, G.; Lakshmi, G.; Ravindranath, N.H. Perspective: Jatropha cultivation in southern India: Assessing farmers' experiences. Biofuels Bioprod. Biorefin. 2012, 6, $246-256$. [CrossRef]

9. SAT-BBE. Annotated Bibliography on Qualitative and Quantitative Models for Analysing the Bio-Based Economy; Systems Analysis Tools Framework for the EU Bio-Based Economy Strategy: The Hague, The Netherlands, 2014; Deliverable 2.3.

10. MINAGRO. BioEconomía Argentina Visión desde Agroindustria; MINAGRO: Buenos Aires, Argentina, 2016.

11. Wierny, M.; Coremberg, A.; Costa, R.; Trigo, E.; Regúnaga, M. Measuring the Bioeconomy: Quantifying the Argentine Case; Grupo Bioeconomia: Buenos Aires, Argentina, 2015.

12. Bundesministerium für Ernährung und Landwirtschaft (BMEL). Bioeconomy in Germany: Opportunities for a Bio-Based and Sustainable Future; Bundesministerium für Ernährung und Landwirtschaft (BMEL): Berlin, Germany, 2015.

13. Bundesministerium für Ernährung und Landwirtschaft (BMEL). National Policy Strategy on Bioeconomy. Renewable Resources and Biotechnological Processes as a Bais for Food, Industry and Energy; Bundesministerium für Ernährung und Landwirtschaft (BMEL): Berlin, Germany, 2014.

14. Bioeconomy Corporation. Bioeconomy Transformation Program: Enriching the Nation, Securing the Future; Bioeconomy Corporation: Kuala Lumpur, Malaysia, 2016.

15. Bioeconomy Corporation. Bioeconomy Transformation Programme: Enriching the Nation; Bioeconomy Corporation: Kuala Lumpur, Malaysia, 2007.

16. Meesters, K.P.H.; van Dam, J.E.G.; Bos, H.L. Protocol Monitoring Materiaalstromen Biobased Economie; Rijksdienst voor Ondernemend Nederland (RVO): Wageningen, The Netherlands, 2013.

17. CE Delft. Sustainable Biomass and Bioenergy in The Netherlands: Report 2015; CE Delft: Delft, The Netherlands, 2016.

18. The Netherlands Offices for Science and Technology (NOST). The Bio-Based Economy in the Netherlands; The Netherlands Offices for Science and Technology (NOST): The Hague, The Netherlands, 2013.

19. NNFCC-The Bioeconomy Consultants. Bioeconomy Factsheet-Netherlands; NNFCC-The Bioeconomy Consultants: York, UK, 2015.

20. Kwant, K.; Hamer, A.; Siemers, W.; Both, D. Monitoring Biobased Economy in Nederland 2016; Rijksdienst voor Ondernemend Nederland (RVO): Wageningen, The Netherlands, 2017. 
21. European Commission. Bioeconomy Knowledge Centre. Bioeconomy Data Catalogue. Available online: https:/ / data-bioeconomy.jrc.ec.europa.eu/ (accessed on 4 April 2018).

22. Public Understanding of Biotechnology (PUB). South Africa Launches its Bio-Economy Strategy; The South African Agency for Science and Technology Advancement: Pretoria, South Africa, 2014.

23. Department of Science and Technology. The Bio-Economy Strategy; Department of Science and Technology: Pretoria: South Africa, 2013.

24. NACI Council. NACI Annual Report 2016/17; NACI Council: Pretoria, South Africa, 2017.

25. The White House. National Bioeconomy Blueprint; The White House: Washington, DC, USA, 2012.

26. U.S. Department of Energy. 2016 Billion-Ton Report: Advancing Domestic Resources for a Thriving Bioeconomy, Volume 1: Economic Availability of Feedstocks; U.S. Department of Energy: Washington, DC, USA, 2016; Volume 1160.

27. United States Department of Agriculture (USDA). Biobased Economy Indicators; United States Department of Agriculture (USDA): Washington, DC, USA, 2011.

28. United States Department of Agriculture (USDA). An Economic Impact Analysis of the U.S. Biobased Products Industry: A Report to the Congress of the United States of America; United States Department of Agriculture (USDA): Washington, DC, USA, 2015; Volume 11.

29. United States Department of Agriculture (USDA). An Economic Impact Analysis of the U.S. Biobased Products Industry: 2016 Update; United States Department of Agriculture (USDA): Washington, DC, USA, 2016.

30. U.S. Department of Energy. The U.S. Bioeconomy by the Numbers 2017; U.S. Department of Energy: Washington, DC, USA, 2017; Volume 1.

31. Van Esch, J.W.J. Personal Communication. Questionnarie: Assessing Bioeconomy Contribution to Countries' Economy; Dutch Ministry of Economic Affairs and Climate Policy: The Hague, The Netherlands, 2018.

32. Che Dir, Z. Personal Communication. Questionnarie: Assessing Bioeconomy Contribution to Countries' Economy; Bioeconomy Corporation: Kuala Lumpur, Malaysia, 2018.

33. Durham, B. Personal Communication. Questionnarie: Assessing Bioeconomy Contribution to Countries' Economy; Department of Science and Technology: Pretoria, South Africa, 2018.

34. SAT-BBE. Tools for Evaluating and Monitoring the EU Bioeconomy: Indicators; Systems Analysis Tools Framework for the EU Bio-Based Economy Strategy: The Hague, The Netherlands, 2013; Deliverable 2.2.

35. The European Innovation Partnership 'Agricultural Productivity and Sustainability' (EIP-AGRI). EIP-AGRI Workshop "Opportunities for Agriculture and Forestry in the Circular Economy"; Workshop Report; European Commission: Brussels, Belgium, 2015.

36. Bioeconomy Corporation. Analysing the Contribution of Malaysian Bioeconomy Using the GDP Approach; Bioeconomy Corporation: Kuala Lumpur, Malaysia, 2015.

37. Ronzon, T.; Piotrowski, S.; M'Barek, R.; Carus, M. A systematic approach to understanding and quantifying the EU's bioeconomy. Bio-based Appl. Econ. 2017, 6, 1-17. [CrossRef]

38. Von Braun, J. Bioeconomy and sustainable development-Dimensions. Rural 2014, 21, 6-9.

39. PBL. Sustainability of Biomass in a Bio-Based Economy; PBL Netherlands Environmental Assessment Agency: The Hague, The Netherlands, 2012.

40. CE Delft. Sustainable Biomass and Bioenergy in The Netherlands: Report 2016; CE Delft: Delft, The Netherlands, 2017.

41. Schachtsiek, T. Personal Communication. Questionnarie: Assessing Bioeconomy Contribution to Countries' Economy; Federal Ministry for Agriculture and Food (BMEL): Bonn, Germany, 2018.

42. Al-Amin, A.Q. Developing a Measure for Quantifying Economic Impacts: The Bioeconomy Contribution Index; Bioeconomy Corporation: Kuala Lumpur, Malaysia, 2015.

43. Golden, J.S.; Handfield, R.B. Why Biobased? Opportunities in the Emerging Bioeconomy; US Department of Agriculture, Office of Procurement and Property Management: Washington, DC, USA, 2014.

44. Rogers, J.N.; Stokes, B.; Dunn, J.; Cai, H.; Wu, M.; Haq, Z.; Baumes, H. An assessment of the potential products and economic and environmental impacts resulting from a billion ton bioeconomy. Biofuels Bioprod. Biorefin. 2017, 11, 110-128. [CrossRef]

45. Nattrass, L.; Biggs, C.; Bauen, A.; Parisi, C.; Rodríguez-Cerezo, E.; Gómez-Barbero, M. The EU Bio-Based Industry: Results from a Survey; Joint Research Centre (JRC) Technical Report; Joint Research Centre: Brussels, Belgium, 2016; Volume EUR 27736 EN. [CrossRef] 
46. Ronzon, T.; Lusser, M.; Landa, L.; M’Barek, R.; Giuntoli, J.; Cristobal, J.; Parisi, C.; Ferrari, E.; Marelli, C.; Torres de Matos, C.; et al. Bioeconomy Report 2016; Klinkenberg, M., Sanchez Lopez, J., Hadjamu, G., Belward, A., Camia, A., Eds.; Joint Research Centre (JRC) Scientific and Policy Report; Joint Research Centre: Brussels, Belgium, 2017; Volume EUR 28468.

47. Mulder, M. National Biotech Survey; Egolibio: Pretoria, South Africa, 2003.

48. Johnson, F.X. Biofuels, Bioenergy and the Bioeconomy in North and South. Ind. Biotechnol. 2017, 13, $289-291$. [CrossRef] [PubMed]

49. European Commission. Jobs and Turnover in the European Union Bioeconomy. European Commission: EU Sciente Hub, DataM. 2018. Available online: https://datam.jrc.ec.europa.eu/datam/mashup/ BIOECONOMICS/index.html (accessed on 21 May 2018).

50. Ekins, P.; Hughes, N.; Bringezu, S.; Clarke, C.A.; Fischer-Kowalski, M.; Graedel, T.; Hajer, M.; Hashimoto, S.; Hatfield-Dodds, S.; Havlik, P.; et al. Resource Efficiency: Potential and Economic Implications. A Report of the International Resource Panel; United Nations Environment Programme: Nairobi, Kenya, 2016; ISBN 9789280736458.

51. Preston, F.; Lehne, J. A Wider Circle? The Circular Economy in Developing Countries; Chatham House, The Royal Institute of International Affairs: London, UK, 2017; ISBN 9781784132569.

52. US Biomass R\&D Board. Federal Activities Report on the Bioeconomy; US Biomass R\&D Board: Washington, DC, USA, 2016.

53. Staffas, L.; Gustavsson, M.; McCormick, K. Strategies and policies for the bioeconomy and bio-based economy: An analysis of official national approaches. Sustainablity 2013, 5, 2751-2769. [CrossRef]

54. Van den Bergh, J.C.J.M. Abolishing GDP. SSRN Electron. J. 2007. [CrossRef]

55. Finnish Ministry of Employment and the Economy. The Finnish Bioeconomy Strategy; Finnish Ministry of Employment and the Economy: Helsinki, Finland, 2014.

56. Presidency of Council of Ministers. Bioeconomy in Italy; Presidency of Council of Ministers: Roma, Italy, 2017.

57. Stockholm Environment Institute. Re-linking Objectives and Potentials; Stockholm Environment Institute: Stockholm, Sweden, 2018.

58. Natural Resources Institute Finland (LUKE). MontBioeco-Synthesis on Bioeconomy Monitoring Systems in the EU Member States; LUKE: Helsinki, Finland, 2018.

59. Fielding, M.; Aung, M.T. Bioeconomy in Thailand: A Case Study; Stockholm Environment Institute: Stockholm, Sweden, 2018.

60. Lewandowski, I.; Faaij, A.P.C. Steps towards the development of a certification system for sustainable bio-energy trade. Biomass Bioenergy 2006, 30, 83-104. [CrossRef] 